\title{
Research on the Flexural Deformation of the Damping Valve Slice
}

\author{
Yijie Chen, Yafeng Zhang, Mengyan Xu, Fu Du, Peng Gui
}

China North Vehicle Research Institute, Beijing 100072, China

chenyijie1206@163.com

*corresponding author: Yijie Chen

Keywords: damping valve, annular valve slice, nonuniform load, flexural deformation.

\begin{abstract}
The damping valve structure of shock absorber usually takes throttle slice as the main part, when the pressure difference at both ends of the valve and the pretightening force of the valve slice can be overcome, annular gap will be formed between the valve body and slice to form throttle channel, so as to achieve the purpose of attenuating external vibration. In this case, the valve slice mechanical model is established, and the deflection formula under the nonuniform load is deduced, which can calculated the deformation of valve slice at any position, and the analysis of the influence factors is carried out, which lays a foundation for study the performance characteristics of the damping device.
\end{abstract}

\section{Introduction}

There are many methods to produce damping force in the shock absorbers of vehicle suspension, and one of the methods which oil is throttled through deformation aperture of valve slice is widely used in engineering practice. It is easier for processing and assembling, and its cost is lower. Because the deflection of slice has great influence on damping force, it is necessary to calculate accurately, but most of the current methods are approximate calculation, and it can only get the outside edge deformation when the slice is forced by uniform distribution of load. ${ }^{[1]}$ There is no suitable analytical equation to offer reference when the valve slice is affected by uniform distribution of variety load. So if people encounter the uniform distribution of variety load acted on the surface of valve slice, they have no choice but to use uniform distribution of load to replace it. ${ }^{[2]}$ However in practice, it has indicated that the deviation is obvious in this way, and it is difficult to make the ideal data of damping force keep coherent with test. In this condition, the deformation analytical equation is deduced with the theory of sheet mechanics in this paper when the slice is forced by the uniform distribution of variety load, and the corresponding systems analysis has been done.

\section{Physical Model of Valve Slice}

As is shown in figure 1, it is the schematic diagram when the throttle valve is opened. The slice is mounted outside of the piston end, which belongs to the structure of unilateral valve. During the piston moves, if the pressure of above cavity is larger than the below cavity, there will form annular aperture between the slice and the end of the piston to throttle the oil and produce damping force in order to reduce the vehicle vibration when the slice is bended. Because of the influence of the aperture, the valve slice will get the uniform distribution of variety load after the valve is opened.

The valve slice model is simplified as is shown in figure 2 . The inside diameter of slice is totally restricted and the outside diameter is free. In the figure, $q$ is the local uniform distribution of load, $q_{1}$ is the edge load of slice, $h$ is the thickness of slice, $r_{a}$ is the inside radius of slice, $r_{b}$ is the outside radius of slice, $r_{c}$ is the effecting radius of local uniform distribution of load. 

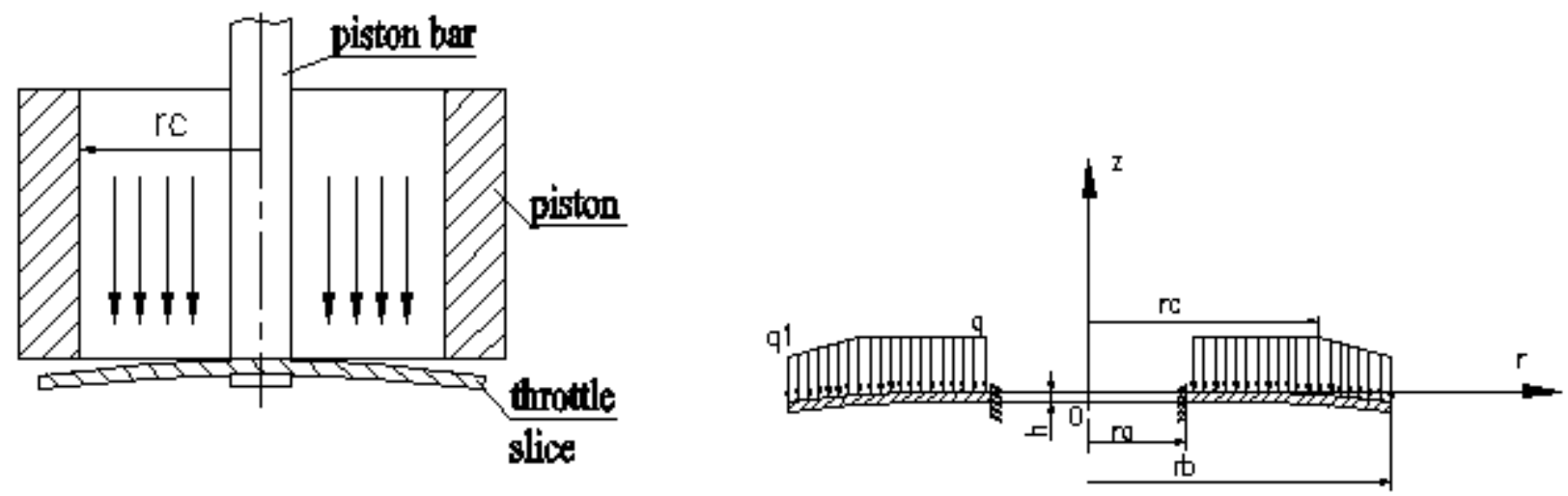

Figure 1 The schematic diagram of throttle valve. Figure 2 The physical model of valve slice.

\section{Deflection Equation of Valve Slice}

According to the sheet mechanics, the differential equation of deformation potential energy of slice in the polar coordinates is as: ${ }^{[3]}$

$$
U=\frac{D}{2} \iint\left[r\left(\frac{d^{2} w}{d r^{2}}\right)^{2}+\frac{1}{r}\left(\frac{d w}{d r}\right)^{2}+2 u \frac{d w}{d r} \frac{d^{2} w}{d r^{2}}\right] d r d \theta
$$

In the equation, $D=\frac{E h^{3}}{12\left(1-u^{2}\right)}$ is the deformation stiffness of slice, $r$ is the radius of slice, $\theta$ is the circumferential angle of slice, $w$ is the deflection of slice, $u$ is poisson's ratio.

As is shown in figure 2, when $r \leq r_{c}$, the load which acts on annular slice is:

$$
Q=q
$$

When $r_{c} \leq r \leq r_{b}$, the expression of load which effect on annular slice is deduced as:

$$
Q=\frac{q-q_{1}}{r_{c}-r_{b}}\left(r-r_{c}\right)+q
$$

The equation of the energy which is acted on the slice by external force is:

$$
W=\iint Q w r d r d \theta
$$

The equation of total potential energy is deduced when the slice is axis symmetry through the equations (1) and (4) ${ }^{[4]}$ :

$$
\Pi=\pi D \int\left[r\left(\frac{d^{2} w}{d r^{2}}\right)^{2}+\frac{1}{r}\left(\frac{d w}{d r}\right)^{2}+2 u \frac{d w}{d r} \frac{d^{2} w}{d r^{2}}\right] d r-2 \pi \int Q w r d r
$$

The deflection equation of slice is expressed through progression as follows:

$$
w(r)=\sum_{m} C_{m} w_{m}(r) \quad m=1,2,3 \cdots
$$

In the equation, $C_{m}$ is unknown coefficient and the serial number is $m, w_{m}(r)$ is carrier function. Every monomial of the equation (6) should be limited by the geometry boundary conditions of slice.

According to the theory of minimal energy, the variation equation of expression (5) is deduced as follows: 


$$
\frac{\partial U}{\partial C_{m}}=2 \pi \int q w_{m}(r) r d r
$$

The boundary conditions of valve slice is given as following according to figure $2:{ }^{[5]}$

$$
\left.w\right|_{r=r_{a}}=0 ;\left.\quad \frac{d w}{d r}\right|_{r=r_{a}}=\left.0 \quad M\right|_{r=r_{b}}=0 ;\left.V\right|_{r=r_{b}}=0
$$

In the equation, $M$ is bending moment of slice, $V$ is total distribution shear stress of slice.

Considering the boundary conditions of equation (8) and the equation (6), we choose the deflection expression of slice as follows:

$$
w=\left(1-\frac{r^{2}}{r_{a}^{2}}\right)^{2}\left[C_{1}+C_{2}\left(1-\frac{r^{2}}{r_{a}^{2}}\right)+C_{3}\left(1-\frac{r^{2}}{r_{a}^{2}}\right)^{2}\right]
$$

Combining the equations (8)and (9) to deduce the expressions of $C_{1}$ and $C_{2}$ which are about $C_{3}$, and take them back to equation (9), then only $C_{3}$ is unknown coefficient in the expression of $w$. The first derivative of $w$ to the radius $r$ of slice is given by:

$$
\frac{d w}{d r}=-\frac{8 C_{3} r\left(r_{a}^{2}-r^{2}\right)}{r_{a}^{8} A_{1}}\left(A_{2}+A_{3}\right)
$$

Where,

$$
\begin{gathered}
A_{1}=r_{a}^{2}(1+u)\left(r_{a}^{2}-3 r_{b}^{2}\right)+2 r_{b}^{4}(2+u) \\
A_{2}=2 r_{b}^{4}(u+2)\left(r_{a}^{4}+r^{4}\right)-r_{b}^{6}(11+5 u)\left(r_{a}^{2}+r^{2}\right) \\
A_{3}=r_{a}^{2}(u+1)\left(r_{a}^{2} r^{4}-3 r_{b}^{2} r_{a}^{2} r^{2}-3 r_{b}^{2} r^{4}\right)+2 r_{b}^{4} r_{a}^{2} r^{2}(4 u+5)+3 r_{b}^{8}(3+u)
\end{gathered}
$$

And the second derivative of $w$ to the radius $r$ of slice is given by:

$$
\frac{d^{2} w}{d r^{2}}=-\frac{8 C_{3}}{r_{a}^{8} A_{1}}\left\{B_{1}+B_{2}\right\}
$$

Where,

$$
\begin{gathered}
B_{1}=2 r_{b}^{4}(u+2)\left(r_{a}^{6}-7 r^{6}\right)+r_{b}^{6}(5 u+11)\left(5 r^{4}-r_{a}^{4}\right)+3 r_{b}^{8}(u+3)\left(r_{a}^{2}-3 r^{2}\right) \\
B_{2}=r^{2} r_{a}^{2}(1+u)\left[3 r_{b}^{2}\left(7 r^{4}-3 r_{a}^{4}\right)+r_{a}^{2}\left(18 r_{b}^{4}-7 r^{4}\right)+5 r^{2}\left(r_{a}^{4}-6 r_{b}^{4}\right)\right]
\end{gathered}
$$

Combining the equations (1), (10) and (14), the results is obtained as follows:

$$
U=-\frac{128 \pi D C_{3}{ }^{2}\left(r_{a}{ }^{2}-r_{b}{ }^{2}\right)^{5}}{35 r_{a}^{16} B_{3}{ }^{2}}\left(B_{4}+B_{5}+B_{6}+B_{7}\right)
$$

Where,

$$
\begin{aligned}
& B_{3}=r_{a}^{4}(1+u)+2 r_{b}^{4}(2+u)-3 r_{a}^{2} r_{b}^{2}(1+u) \\
& B_{4}=3 r_{a}^{12}\left(1+u^{2}+2 u\right)-24 r_{a}^{10} r_{b}^{2}\left(u^{2}+1+2 u\right)
\end{aligned}
$$




$$
\begin{gathered}
B_{5}=8 r_{a}^{8} r_{b}^{4}\left(13+8 u^{2}+21 u\right)-2 r_{a}^{6} r_{b}^{6}\left(31 u^{2}+117+148 \mathrm{u}\right) \\
B_{6}=r_{a}^{4} r_{b}^{8}\left(313+25 u^{2}+158 u\right)-2 u r_{a}^{2} r_{b}^{10}(9 u-39) \\
B_{7}=6 r_{b}^{12}\left(15+2 u^{2}+15 u\right)
\end{gathered}
$$

The common factors are picked up and the deflection expression of the annular valve slice is reorganized as follows:

$$
w=\frac{G_{L}(r)}{E h^{3}}\left(q X+q_{1} Y\right)
$$

Where, $G_{L}$ is the deformation coefficient of uniform distribution of variety load, $E$ is the elasticity modulus of material.

Because there are no presented equations currently related to the annular slice under the uniform distribution of variety load, the correctness of the equation (23) is validated by using the finite element method.

Firstly, the related parameters are assumed as follows: the inside radius $r_{a}=27.5 \times 10^{-3} \mathrm{~m}$, the outside radius $r_{b}=40 \times 10^{-3} \mathrm{~m}$, the thickness of slice $h=0.5 \times 10^{-3} \mathrm{~m}$, the local uniform distribution of load $q=0.2 \mathrm{Mpa}$, the edge load of slice $q_{1}=0.1 \mathrm{Mpa}$, the effecting radius $r_{c}=30 \times 10^{-3} \mathrm{~m}, 35 \times 10^{-3} \mathrm{~m}$, the elasticity modulus $E=2.06 \times 10^{11} \mathrm{~Pa}$,the Poisson's ratio $u=0.3$. The results are shown in figure 3 and 4.

Again, the local uniform distribution of load is changed as $q=0.1 \mathrm{Mpa}, 0.4 \mathrm{Mpa}$, the edge loads are $q_{1}=0.05 \mathrm{Mpa}, 0.2 \mathrm{Mpa}$, the effecting radius is $r_{c}=33 \times 10^{-3} \mathrm{~m}$, and the other parameters are the same as above. The results are shown in figure 5 and 6.

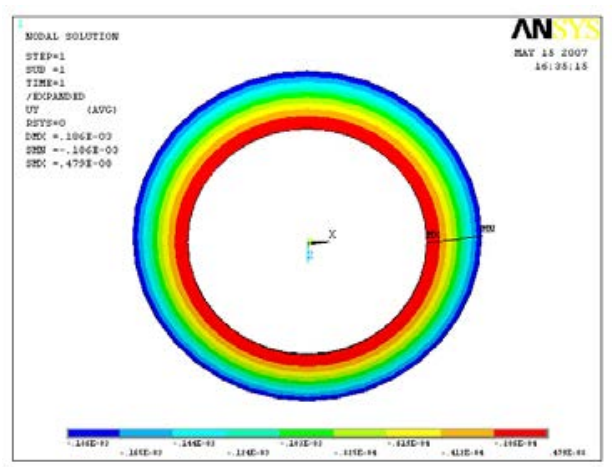

Figure 3 The effecting radius is $30 \times 10^{-3} \mathrm{~m}$.

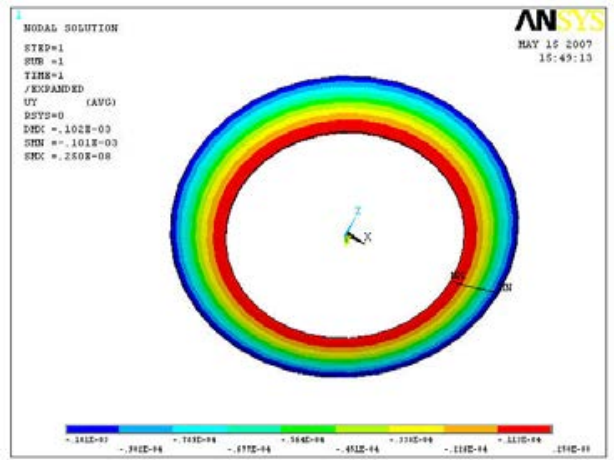

Figure 5 The edge load is $0.05 M p a$.

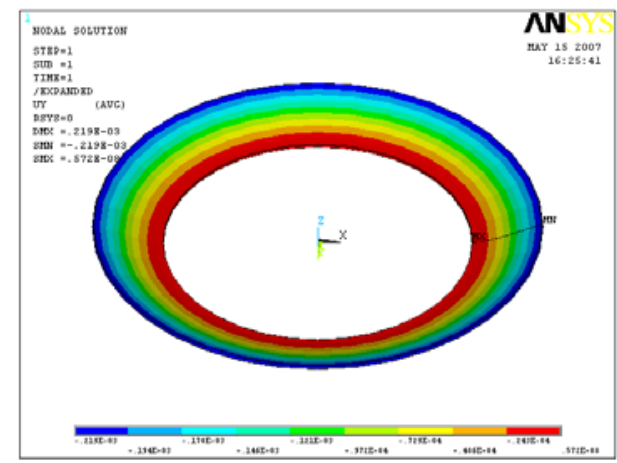

Figure 4 The effecting radius is $35 \times 10^{-3} \mathrm{~m}$.

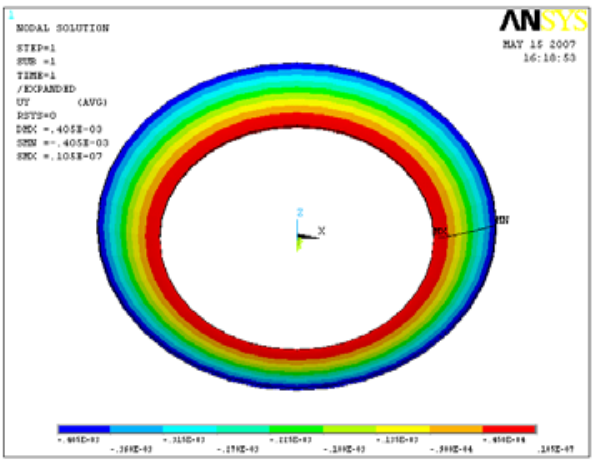

Figure 6 The edge load is $0.2 \mathrm{Mpa}$. 
The outside diameter deflection obtained from the software Ansys and the calculation results of the equation (23) are shown in table 1 and 2.

Table 1 The outside edge deflection in different load effecting radius.

\begin{tabular}{|c|c|c|c|}
\hline$r_{c} / m$ & $f_{A} / m$ & $f / m$ & $\Delta f / \%$ \\
\hline $30 \times 10^{-3}$ & $0.186 \times 10^{-3}$ & $0.1831 \times 10^{-3}$ & 1.56 \\
\hline $35 \times 10^{-3}$ & $0.219 \times 10^{-3}$ & $0.2158 \times 10^{-3}$ & 1.46 \\
\hline
\end{tabular}

Table 2 The outside edge deflection in different edge load.

\begin{tabular}{|c|c|c|c|}
\hline$q_{1} / M p a$ & $f_{A} / m$ & $f / m$ & $\Delta f / \%$ \\
\hline 0.05 & $0.101 \times 10^{-3}$ & $0.0999 \times 10^{-3}$ & 1 \\
\hline 0.2 & $0.405 \times 10^{-3}$ & $0.3995 \times 10^{-3}$ & 1.3 \\
\hline
\end{tabular}

In the tables, $f_{A}$ is the outside diameter deflection of Ansys, $f$ is the outside diameter deflection of equation (23), and $\Delta f$ is the relative error between them.

The simulation results are shown in figure 7-9, it is shown that with the increase of the effecting radius $r_{c}$, the edge load $q_{1}$ and the local uniform distribution of load $q$, the deflection of valve slice at any radius increase respectively.
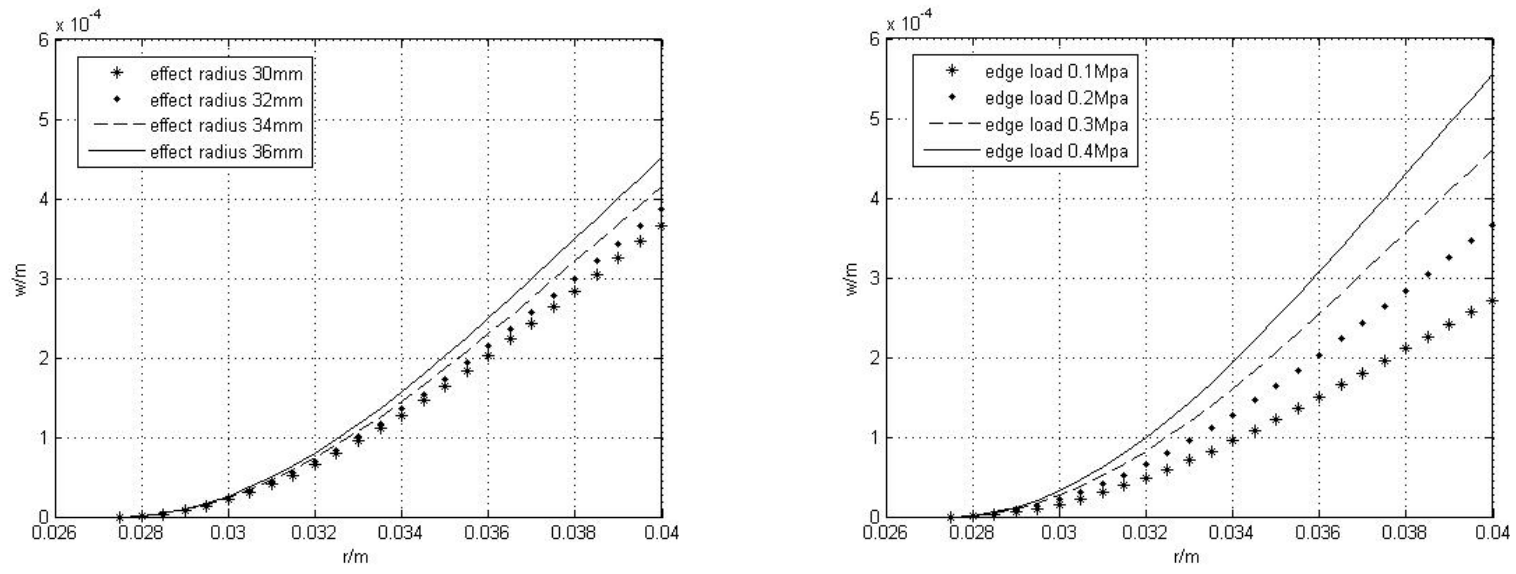

Figure 7 The results in different load effecting radius. Figure 8 The results in different edge loads.
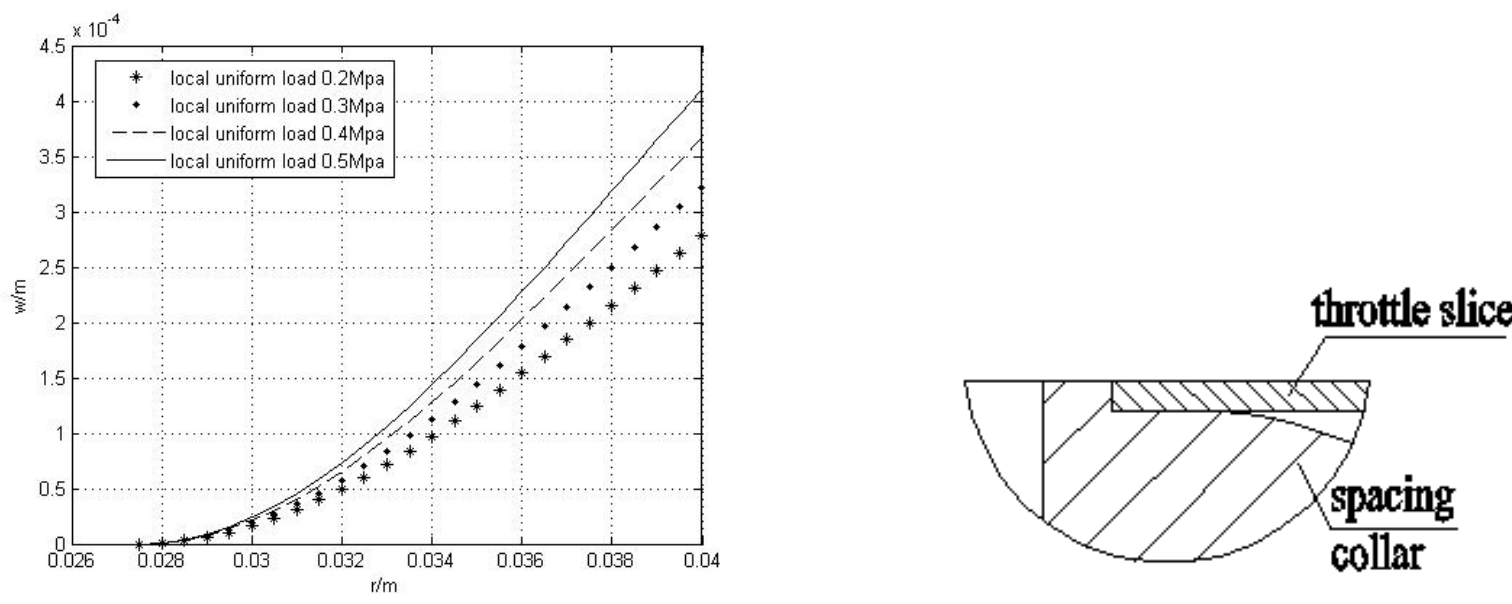

Figure 9 The results in different local uniform loads. Figure 10 Valve slice and spacing collar.

In addition, the formula (23) also can be used to design accurate spacing collar contour (shown in figure 10) to limit the maximum deformation of the slice at any radius in order to avoid stress 
concentration inducing the slice fatigue break when excessive deformation happens.

\section{Conclusions}

The physical model of the slice is built according to the typical assembly mode of the throttle valve of shock absorber, and the concept of uniform distribution of variety load is presented. The deflection analytical equation under uniform distribution of variety load is deduced. The deduced equation can calculate the deflection of the slice at any radius accurately. The conclusions are drawn as follows through the simulation and analysis:

(1) The numerical values of finite element model are contrasted with the analytical results of the equation (23) at different edge loads and effecting radius. Because the deviation of them is very small, the correctness of analytical equation deduced is validated.

(2) The rules that the parameters of slice structure and material to the deflection are studied. It offers reference to the research of deflection of the annular slice.

(3) The deflection of slice at any radius can be calculated with the analytical equation (23) under given load. It provides the theoretical foundation for designing the spacing collar accurately.

\section{References}

[1] Cheng Daxian. Mechanical design handbook the first book. Bei Jing Chemic Industry publishing house. 2002, p1-168-6.

[2] Hu Yuanbing. Design and calculation of elastic valve block for shock absorber valve train. Design Test Study.2002, 5. p13- 15.

[3] Duflou, J.R; Nguyen, T.H.M; Kruth, J.P. Geometric reasoning for tool selection in sheet metal bending operations. International Conference on Integrated Design and Manufacturing in Mechanical Engineering (IDMME 2004), 2004, p15.

[4] Hirota, K; Mori, Y. Precision small angle bending of sheet metals using shear deformation. JSME International Journal, Series A (Solid Mechanics and Material Engineering), v 48, n 4, Oct. 2005, p352-7.

[5] Alinger, M. J; Van Tyne, C. J. Evolution of die surfaces during repeated stretch-bend sheet steel deformation. Journal of Materials Processing Technology, v141, n3, Nov1, 2003, p411-419. 